\title{
ASiT's concerns about views expressed in the Nuffield Trust report on emergency general surgery
}

An error was introduced by $T h e B M J$ in the second paragraph of this letter (BMJ 2016;353:i2593, doi:10.1136/bmj.i2593) during the editing process. The second sentence of this paragraph ("Importantly, the report does not state that only two of the 155 trainees studied had a special interest") should have said that the Nuffield Trust report on emergency general surgery does not state that only two of the 155 trainees studied had general surgery as their special interest. The remaining 153 trainees declared a specialist interest within general surgery, for example, colorectal surgery. We apologise for this error.

See this rapid response for a more detailed explanation http:// www.bmj.com/content/353/bmj.i2593/rr 